\title{
Mise en œuvre d'interventions sociales en soins de santé primaires
}

\author{
Gary Bloch MD, Linda Rozmovits DPhil
}

Citation : CMAJ 2021 November 8;193:E1696-1701. doi : 10.1503/cmaj.210229-f

Voir la version anglaise de l'article ici : www.cmaj.ca/lookup/doi/10.1503/cmaj.210229

D e nos jours, la plupart des médecins de famille déclarent intégrer un certain niveau d'intervention sociale dans la prise en charge de leurs patients ${ }^{1}$. Cependant, à l'exception des centres de santé communautaires, les interventions sociales ne font toujours pas partie de la pratique de routine des soins de santé primaires et ne sont pas encore considérées comme la «norme thérapeutique ». Jusqu'au début des années 1990, les soins de santé primaires traditionnels comprenaient rarement des interventions comme la prescription sociale et des partenariats médico-juridiques ${ }^{2}$ et certains praticiens s'interrogent toujours sur le rôle du professionnel en soins de santé primaires dans l'intervention sociale ${ }^{3}$. Un petit noyau de praticiens issus de pays à revenu élevé, dont le Canada, l'Australie, le Royaume-Uni et les États-Unis, a été à l'avant-garde de l'élaboration et de l'évaluation d'interventions en matière de risques sociaux pour la santé qui ont mené à ce que quelques interventions sociales soient adoptées à grande échelle, avec des répercussions positives sur de vastes marqueurs de la santé.

Il est difficile de produire des travaux de recherche de haute qualité qui trouvent leur application en clinique tout en menant à des interventions sociales en soins de santé primaires. Les effets d'une vie soumise à des pressions sociales comme la pauvreté, le racisme ou des traumatismes sont difficiles à évaluer à l'aide des marqueurs de changement traditionnels en santé physique ou mentale sur l'intervalle de temps d'une étude typique. Pour cette raison, la documentation sur les interventions sociales en soins de santé primaires se concentre souvent sur le processus plutôt que sur la mesure des retombées, de même que sur des indicateurs de santé et de bien-être autodéclarés ${ }^{31}$. Malgré ces limites, la documentation démontre des répercussions généralement positives des interventions sociales sur la santé.

Nous discutons des données probantes amassées (encadré 1 ) sur les interventions sociales et nous présentons un survol des interventions courantes basées sur les soins de santé primaires (tableau 1), soulignant les forces, les limites et la faisabilité de la mise en œuvre dans différents milieux de pratiques. Nous discutons des niveaux de ressources qui faciliteraient leur mise en œuvre et nous suggérons aussi des interventions qui pourraient mener à la création de partenariats avec la communauté externe, ce qui pourrait offrir une alternative aux établissements

\section{Points clés}

- Les interventions sociales basées sur les soins de santé primaires offrent un important moyen d'atténuer les menaces posées par les conditions sociales défavorables à la santé des personnes et des communautés.

- Parmi les interventions efficaces, on compte celles qui ciblent les déterminants au niveau individuel, les liaisons avec les ressources communautaires, les partenariats centrés sur la communauté et les structures au sein des équipes de santé qui influent sur l'équité.

- De plus en plus de données probantes indiquent des répercussions positives des interventions sociales sur de vastes marqueurs de la santé; cependant, la plupart des travaux de recherche dans ce domaine se sont concentrés sur la mesure de la mise en œuvre et du processus, plutôt que sur les résultats.

- Certaines interventions nécessitent de vastes ressources interdisciplinaires de soins de santé pour procéder à la mise en œuvre, mais plusieurs sont accessibles à de petits cabinets de groupe ou à des fournisseurs de soins qui exercent seuls.

moins bien pourvus. Bien que l'intégration de nouvelles interventions peut sembler intimidante, particulièrement pour les professionnels de la santé établis dans la communauté sans le soutien d'une équipe interdisciplinaire, nous avons pris part à des processus de changement auprès d'établissements de toutes tailles pour y intégrer les interventions sociales.

\section{Comment peut-on cibler les besoins sociaux des patients?}

Afin d'aborder les facteurs de risques sociaux pour une mauvaise santé, la première étape est de cibler les besoins d'une personne en lien avec les facteurs sociaux qui pourraient avoir une incidence sur sa santé, comme le revenu, le logement, la littératie, l'éducation et la situation d'emploi, ou des expériences de vie socialement marquantes, comme un traumatisme, le racisme, l'homophobie ou la violence conjugale. Les questions liées aux besoins sociaux permettent aussi de relever les ressources individuelles et communautaires qui pourraient être exploitées dans la mise en place d'un programme de soins, comme des réseaux de soutien ou des associations de voisinage. 
Encadré 1 : Données probantes employées dans cette revue

En octobre 2018, avec l'aide d'une bibliothécaire possédant une expertise en recherche médicale, nous avons réalisé une vaste revue documentaire non systématique, que nous avons mise à jour en novembre 2020. Nous avons fouillé les bases de données MEDLINE, Embase, CINAHL et Sociological Abstracts. Les termes de recherche, qui variaient légèrement selon la base de données, comprenaient " *primary health care/or patient-centered care", " primary care », " physicians, family », " general pract* », " family pract* », " family physician* », « social determinants of health », " social equit* ", " social screening », " social prescribing ", " social determinant" » et " social barrier* " [" "soins de santé primaires/ou soins centrés sur le patient ", " soins de santé primaires », " médecins, famille », "médecin * traitant ", "médecin* traitant de famille ", " médecin* de famille », "déterminant sociaux de la santé ", "équité* sociale ", "dépistage social ", "prescription sociale », " déterminant* social » et " barrière* sociale »]. Nous avons limité la recherche aux articles de langue anglaise. Nous avons relevé la littérature grise au moyen de discussions avec des experts, en examinant des listes de références et en procédant à des recherches en ligne à l'aide de Google. Dans l'optique de garder notre attention sur des interventions reproductibles dans l'ensemble des milieux de pratique, nous avons limité notre recherche aux pays à revenu élevé. Cette recherche a produit 895 résumés indépendants, que nous avons tous les deux révisés pour en déterminer la pertinence. Nous avons sélectionné 177 articles pour un examen complet.

\section{Outils de dépistage}

Consigner d'emblée les pressions sociales vécues par un patient lors des consultations médicales permet au professionnel de la santé d'adapter son évaluation des besoins et de déterminer les ressources requises pour y répondre. Le dépistage des besoins sociaux est aussi un prérequis pour que les patients aient accès aux programmes de prestations et aux services des organismes communautaires.

Les outils actuellement disponibles vont de ceux qui soutiennent des approches de dépistage ciblées pour des déterminants uniques, comme le revenu ou les expériences défavorables vécues pendant l'enfance, à ceux qui fournissent une évaluation plus complète des besoins sociaux. L'outil «Pauvreté : un outil clinique pour les prestataires de soins primaires » offre une seule question de dépistage en lien avec la sécurité du revenu : "Avez-vous parfois de la difficulté à boucler votre budget à la fin du mois? " Simple à utiliser, cette question présente une sensibilité de $98 \%$ et une spécificité de $40 \%$ pour cibler les personnes vivant sous le seuil de la pauvretét,5. Des outils de dépistage des besoins sociaux plus complets, comme HealthBegins et le Protocol for Responding to and Assessing Patients' Assets, Risks and Experiences (PRAPARE - Protocole pour répondre et évaluer les ressources, les risques et les expériences vécues des patients), explorent de multiples domaines de risques sociaux qui vont du logement aux services de garde d'enfants en passant par la sécurité du voisinage $e^{6}$. L'évaluation d'outils à volets multiples a démontré que plusieurs personnes présentent des besoins sociaux multiples qui se recoupent ${ }^{7}$. Le réseau Siren a publié un tableau comparatif des outils de dépistage social utilisés aux États-Unis ${ }^{8}$.
La mise en œuvre du dépistage nécessite de la formation et l'adhésion des professionnels de la santé en première ligne. Elle requière aussi un engagement soutenu et l'attribution de temps et de ressources ${ }^{9-11}$. Cependant, ni la formation ni les demandes de ressources ne sont coûteuses. Certains outils de dépistage peuvent être mis en œuvre par le personnel administratif ou à l'aide d'interfaces électroniques, comme une tablette à présenter aux patients afin qu'ils remplissent de simples questionnaires de façon autonome ${ }^{12,13}$.

\section{Recherche locale}

Bien que l'objectif principal des outils de dépistage cliniques soit de cibler les besoins individuels, les données au niveau de la pratique et de la population peuvent engendrer des efforts de plus haut niveau pour participer aux interventions sociales. Les données obtenues des dossiers médicaux électroniques peuvent être employées à dresser un portrait des besoins sociaux au sein d'une population désservie ${ }^{14}$ et de guider la qualité des initiatives d'amélioration ${ }^{15}$. De cette façon, le dépistage des besoins sociaux peut former une assise pour poursuivre les actions sur les risques sociaux sur la santé et éclairer la conception, le suivi et l'évaluation des interventions.

Le projet HealtheRx de l'Université de Chicago et le site Web Ontario Health Profiles interprètent des données sociales de niveau communautaire pour qu'elles soient utilisées par des équipes de santé et d'autres services sociaux ${ }^{16,17}$. Le projet University of Toronto Practice-Based Research Network (UTOPIAN - Réseau de recherche fondé sur la pratique de l'Université de Toronto) permet aux chercheurs d'accéder à des données anonymisées provenant des dossiers médicaux électroniques de 1700 médecins de famille ${ }^{18}$. De vastes bases de données sont aussi utilisées pour intégrer des données sociales dans des algorithmes prédictifs faisant appel à l'intelligence artificielle afin d'identifier et de cibler les services destinés aux personnes et aux groupes présentant d'importants besoins ${ }^{19,20}$.

Cibler les personnes ayant les plus grands besoins sociaux et sanitaires et, par conséquent, les plus importants frais de soins de santé, peut être dommageable si ces données sont employées à restreindre les services ${ }^{21,22}$. De plus, l'identification des besoins particuliers ne se traduit pas toujours par l'adoption d'interventions sociales, ce qui peut indiquer une déconnexion entre l'identification du besoin et la capacité à y répondre ${ }^{23}$.

\section{Comment les médecins peuvent-ils faire le lien entre les patients et des soutiens sociaux efficaces?}

La prescription sociale exploite les ressources individuelles et communautaires dans le but d'améliorer le bien-être, l'autogestion et l'autonomisation des personnes ${ }^{24,25}$. Pour ce faire, elle emploie une approche structurée afin d'orienter les patients vers les soutiens communautaires. En plus de se concentrer sur les facteurs en lien avec le mode de vie, comme la pratique de l'activité physique, plusieurs programmes de prescription sociale cherchent à aborder directement les déterminants sociaux, comme le revenu et le logement. 
Tableau 1 : Interventions sociales en soins de santé primaires selon le type, le milieu, les besoins en matière d'infrastructure et la capacité d'être menées par un partenaire communautaire

\begin{tabular}{|c|c|c|c|c|c|}
\hline Intervention & Infrastructure requise & $\begin{array}{l}\text { Praticien } \\
\text { qui exerce } \\
\text { seul }\end{array}$ & $\begin{array}{l}\text { Petit } \\
\text { cabinet de } \\
\text { groupe }\end{array}$ & $\begin{array}{l}\text { Large groupe } \\
\text { interdisciplinaire }\end{array}$ & $\begin{array}{l}\text { Partenaire } \\
\text { communautaire }\end{array}$ \\
\hline $\begin{array}{l}\text { Dépistage des besoins } \\
\text { sociaux }\end{array}$ & $\begin{array}{l}\text { Professionnel de la santé en première ligne } \\
\text { ou personnel de soutien prêt à participer }\end{array}$ & $x$ & $x$ & $\mathrm{x}$ & \\
\hline Littératie & $\begin{array}{l}\text { Professionnels de la santé en première ligne } \\
\text { prêts à participer; ressources financières } \\
\text { pour se procurer des livres }\end{array}$ & $x$ & $x$ & $\mathrm{x}$ & \\
\hline $\begin{array}{l}\text { Spécialistes de la } \\
\text { sécurité du revenu }\end{array}$ & $\begin{array}{l}\text { Dépistage des besoins sociaux et spécialiste } \\
\text { de la sécurité du revenu rémunéré au sein de } \\
\text { l'équipe ou partenariat avec une institution } \\
\text { spécialisée externe }\end{array}$ & & $x$ & $\mathrm{x}$ & $x$ \\
\hline Prescription sociale & $\begin{array}{l}\text { Dépistage des besoins sociaux, agent de } \\
\text { liaison (financé par le cabinet ou en } \\
\text { partenariat avec un organisme externe) et } \\
\text { directeur de pratique pour coordonner et } \\
\text { superviser l'initiative }\end{array}$ & & $\mathrm{x}$ & $x$ & $x$ \\
\hline $\begin{array}{l}\text { Changement de } \\
\text { pratique orienté vers } \\
\text { l'équité }\end{array}$ & $\begin{array}{l}\text { Professionnels de la santé en première ligne } \\
\text { et membres de l'équipe prêts à explorer les } \\
\text { iniquités structurelles, à suivre une formation } \\
\text { et à poursuivre la discussion; consultant } \\
\text { externe ou guide pour faciliter l'exploration } \\
\text { et le changement de pratique }\end{array}$ & & $\mathrm{x}$ & $x$ & \\
\hline $\begin{array}{l}\text { Partenariat médico- } \\
\text { juridique }\end{array}$ & $\begin{array}{l}\text { Dépistage des besoins juridiques et } \\
\text { partenariat avec une agence juridique; } \\
\text { bureau et fournitures pour le personnel } \\
\text { juridique; directeur de pratique pour } \\
\text { coordonner }\end{array}$ & & & $\mathrm{x}$ & $x$ \\
\hline $\begin{array}{l}\text { Collecte de données } \\
\text { sociales }\end{array}$ & $\begin{array}{l}\text { Accès aux données (dans des dossiers } \\
\text { médicaux électroniques ou des bases de } \\
\text { données externes); traitement des données } \\
\text { et expert en analyse }\end{array}$ & & & $x$ & $x$ \\
\hline $\begin{array}{l}\text { Partenariat } \\
\text { communautaire ou } \\
\text { développement } \\
\text { communautaire }\end{array}$ & $\begin{array}{l}\text { Professionnel de la santé en première ligne et } \\
\text { équipe de direction prêts à participer à des } \\
\text { rencontres communautaires et à adapter } \\
\text { l'infrastructure de l'équipe; travailleurs } \\
\text { spécialisés en santé communautaire ou en } \\
\text { développement communautaire }\end{array}$ & & & $x$ & $x$ \\
\hline
\end{tabular}

Typiquement, la prescription sociale nécessite qu'un professionnel de la santé présente une demande de consultation à une ressource communautaire et à un spécialiste de la mobilisation, nommé agent de liaison. L'agent de liaison rencontre les clients afin d'explorer leur situation et de codéterminer leurs besoins pour ensuite les mettre en contact avec les services locaux appropriés, les accompagnant et les soutenant le long de leur parcours au besoin. Les agents de liaison peuvent être intégrés aux pratiques de soins primaires ou à celles situées dans la communauté. Ils offrent une expertise, une capacité et un soutien durable d'une manière qui est rarement possible aux professionnels de la santé en soins primaires.

La prescription sociale est largement mise en œuvre au Royaume-Uni, maintenant soutenue par le National Health Service; le Réseau de prescription sociale organise les pratiques à l'échelle nationale et régionale ${ }^{26}$. Aux États-Unis, le programme
Health Lead repose sur des étudiants universitaires bénévoles pour faciliter les liens qui soutiennent les programmes ${ }^{27}$. Au Canada, l'Alliance pour des communautés en santé a récemment achevé un projet pilote pour élaborer des programmes de prescription sociale conçus pour les communautés au sein des centres de santé communautaires ${ }^{24}$. À Winnipeg, le modèle financé par le gouvernement, My Health Team (Mon équipe santé), offre l'appui de professionnels de la santé multidisciplinaires, dont des spécialistes du revenu et des intervenants pivots de la communauté, à de petits cabinets de groupe ou des pratiques familiales individuelles. Les services sont mobilisés par les professionnels en soins de santé primaires au moyen d'une interface intégrée au dossier médical électronique qui facilite la communication au sein de l'équipe ${ }^{28,29}$. En l'absence d'un tel soutien externe, la prescription sociale nécessite des ressources financières et humaines et elle est plus facilement mise en œuvre par des équipes interdisciplinaires bien outillées ${ }^{30}$. 
La prescription sociale a montré des résultats prometteurs, dont des améliorations dans de vastes marqueurs de la santé et du bien-être ${ }^{32-35}$. Des travaux de recherche réalisés au RoyaumeUni ont rapporté une amélioration de la résilience, de la santé mentale, de la qualité de vie et une modification réussie des facteurs associés aux habitudes de vie, de même que des liens plus solides entre les services de santé et les services communautaires, une diminution de la prescription de médicaments, une augmentation dans la prise en charge préventive et une réduction de l'utilisation des soins primaires par des patients qui cherchent de l'aide pour répondre à leurs besoins sociaux non comblés ${ }^{25}$. Lorsque les agents de liaison jouent un rôle actif dans la prise en charge des cas, on a observé une amélioration des résultats et une plus grande satisfaction au sein de l'équipe de santé ${ }^{36}$.

\section{Comment peut ont répondre aux déterminants sociaux individuels de la santé?}

\section{Sécurité du revenu}

Les programmes de soins de santé primaires orientés vers des interventions en matière de revenu ont émergé dans certains milieux au cours des 30 dernières années et se sont largement concentrés sur le soutien aux personnes en situation de faible revenu afin qu'elles aient accès aux prestations, que leurs dettes soient consolidées et réduites et que leur littératie financière soit améliorée.

Au Royaume-Uni, depuis le milieu des années 1990, on a mis en place des services offrant des conseils en matière de droit à l'assistance publique. Souvent situés au même endroit que les équipes de santé, ces services s'efforcent de maximiser l'accès des clients aux soutiens de l'assistance sociale. Des évaluations ont démontré que l'accès au service est associé avec une augmentation du revenu ainsi qu'avec une amélioration des indices de bien-être ${ }^{37}$.

Au Canada, des spécialistes du revenu sont intégrés à plusieurs équipes de soins de santé primaires. Ils se concentrent sur l'évaluation de la sécurité du revenu individuelle de même que sur la formation des professionnels de la santé et la promotion des politiques sociales. Les analyses préliminaires de la mise en œuvre et les données administratives sont prometteuses, mais les résultats des études des retombées, y compris un essai randomisé et contrôlé, n'ont pas encore été publiés ${ }^{38}$.

Parmi les autres approches prometteuses, on compte du mentorat financier mené par les pairs, des partenariats entre des organismes médicaux et financiers et l'offre de services de production de déclarations de revenus dans l'enceinte des cliniques. Les évaluations ont montré un effet positif sur le bien-être, de même qu'un important rendement de l'investissement ${ }^{39-41}$.

La mise en place de programmes de soutien au revenu nécessite du financement pour l'intégration de spécialistes ou l'établissement de partenariats avec des organismes communautaires qui possèdent l'expertise et la capacité d'accomplir ce travail. Les milieux de pratique qui possèdent peu de ressources peuvent établir et maintenir une liste des programmes de prestations de revenu et des cliniques d'impôts locales et gratuites où les patients peuvent être orientés.

\section{Besoins juridiques}

Des programmes qui offrent des services juridiques directement aux clients des équipes de santé ont émergé aux États-Unis en 1993. En 2010, plus de 200 hôpitaux et centres de santé communautaires américains participaient à ce type de partenariat ${ }^{42}$. Les partenariats médico-juridiques ont depuis aussi émergé au Canada, au Royaume-Uni et en Australie ${ }^{43}$.

Les fournisseurs de services juridiques peuvent être intégrés directement à l'équipe de soins de santé primaires, être situés sur les lieux de pratique ou être accessibles au moyen d'un processus de demande de consultation. En plus de fournir une assistance juridique individuelle aux clients, ils peuvent participer à la formation des professionnels de la santé et des clients et faire la promotion de changements systémiques au moyen de réformes législatives. Ils sont soutenus par du financement gouvernemental ou privé ainsi que par le biais de partenariats avec des organismes de services juridiques privés et publics ${ }^{42}$.

Child HeLP, un partenariat médico-juridique très performant situé à Cincinnati, a jumelé un service de soins de santé primaires spécialisé en pédiatrie avec un organisme juridique communautaire possédant une expertise auprès des populations socialement marginalisées. Une évaluation du programme a démontré des retombées juridiques positives, centrées fortement sur les préoccupations de revenu et de logement pour les enfants atteints de maladie chroniques ${ }^{44}$.

Les études de mise en œuvre et de résultats des partenariats médico-juridiques proposent un modèle réalisable et à forte incidence dont le potentiel d'amélioration des retombées en santé est très grand ${ }^{45}$. Par contre, ils nécessitent une équipe de santé substantielle, des ressources juridiques avec des partenariats communautaires et sont plus facilement mis en place dans les établissements hébergeant des équipes plus importantes ou avec un partenaire communautaire solide ou encore, avec le soutien du gouvernement.

\section{Littératie}

La littératie est le marqueur du niveau d'instruction qui, à un faible degré, peut être associé à la pauvreté. La littératie est souvent un prérequis à l'accès au marché du travail et à une bonne orientation parmi les services sociaux et de santé. Comme d'autres organismes, l'Académie américaine de pédiatrie fait la promotion de la sensibilisation à la littératie comme un élément essentiel de la pratique en soins de santé primaires ${ }^{46}$.

En 1989, on a créé à Boston une intervention en littératie fondée sur la santé, Reach Out and Read; plus de 6400 établissements l'ont mise en œuvre en Amérique du Nord. Elle forme des professionnels de la santé à fournir du soutien en littératie, à offrir des livres gratuits et à orienter les familles et les jeunes enfants vers des organismes œuvrant en littératie. Les évaluations démontrent des retombées positives en littératie, avec une influence tout particulièrement importante sur les enfants des familles à faibles revenus ${ }^{47}$.

On peut mettre en place cette intervention dans les milieux de pratique aux ressources limitées. Elle nécessite une formation initiale et une forme de parrainage pour l'achat de livres, mais ultimement, elle repose sur les professionnels de la santé de première ligne qui consacrent quelques minutes pour discuter de littératie avec les parents lors des consultations médicales d'un enfant. 


\section{Isolement social}

On a démontré que l'isolement social et la solitude sont des facteurs de risque pour une mauvaise santé et d'un risque accru de décès, particulièrement chez les personnes de plus de 50 ans $^{48}$. La prévalence et les incidences de l'isolement social sur la santé sont magnifiées chez les personnes à faible revenu ou sans logement convenable ${ }^{49}$.

Les interventions peuvent lutter contre l'isolement social de différentes façons, y compris en s'attaquant aux problèmes de mobilité et d'audition, en développant les habiletés sociales, en atténuant le manque d'accès au transport et en s'attaquant aux troubles cognitifs et psychologiques à l'aide de psychothérapies ciblées. La qualité des données probantes est pauvre dans la plupart des cas, la plus grande influence étant associée aux interventions qui augmentent l'activité physique ${ }^{50}$.

\section{Situation d'emploi, logement, transport et sécurité alimentaire}

L'éventail de données probantes en matière d'interventions sociales basées sur les soins de santé primaires et centrées sur la personne est croissant, mais demeure relativement clairsemé. Une revue systématique a trouvé quelques interventions fondées sur les soins de santé primaires qui ciblaient la situation d'emploi, se concentrant pour la plupart sur des patients présentant une grave maladie mentale. Cependant, elle a souligné le potentiel de l'intégration de spécialistes en emploi ou de partenariats avec des organismes communautaires afin d'améliorer les retombées en matière d'emploi pour les patients ${ }^{51}$. Les interventions dans le domaine du logement, bien que percutantes, ont presque exclusivement été étudiées dans des établissements de soins de santé spécialisés qui offrent des services aux personnes qui ont vécu l'ittinérance ${ }^{52}$. On cible le transport à des rendez-vous médicaux comme un déterminant de l'accès aux soins et des interventions peuvent permettre au client d'interagir avec les services de soins de santé $e^{53}$. L'insécurité alimentaire est souvent dépistée en soins de santé primaires, mais puisque l'insécurité alimentaire est indicateur d'un revenu insuffisant, l'utilité des interventions qui sont limitées à l'approvisionnement en nourriture est discutable ${ }^{54-56}$.

\section{De quelle façon les professionnels de la santé se mobilisent-ils avec les communautés pour répondre aux besoins sociaux?}

Cibler les déterminants sociaux dans les communautés, plutôt que chez les personnes, au moyen d'interventions collaboratives fondées sur les soins de santé primaires dans les communautés, offre aussi un fort potentiel pour élargir l'intégration et l'influence des équipes de soins de santé primaires ${ }^{57}$.

Le programme Keeping Infants Nourished and Developing (KIND - Garder les nourrissons bien nourris et assurer leur croissance) de Cincinnati a exploité un partenariat communautaire solide afin de s'attaquer à l'insécurité alimentaire et à d'autres besoins sociaux des enfants vivant en situation de pauvretét 54 . Une revue des données probantes a démontré un rendement positif sur l'investissement pour les partenariats santé-communauté centrés sur le logement, la nutrition, le transport, les modifications à apporter au domicile et à la navigation au sein des services sociaux et de soins de santées .

Les travailleurs spécialisés en santé communautaire, qui sont aussi souvent membres de la communauté, contribuent aux connaissances locales des équipes de santé. En plus de cibler les besoins sociaux, ils peuvent mener à la sensibilisation et à la participation des équipes de santé, avec une attention particulière portée à l'éducation en matière de santé, à la coordination des soins et à la littératie médicale. Un programme réalisé à la Clinique Mayo a posté des travailleurs communautaires de la santé dans des établissements sous la cosupervision d'équipes de santé et de groupes communautaires. Les travailleurs étaient souvent issus de parcours socioéconomiques et culturels semblables à ceux des clients et aidaient les patients à naviguer entre les besoins sociaux et de santé. Le programme a entraîné une diminution des consultations externes, de l'utilisation du service des urgences et des coûts de santés9.

Un modèle de développement communautaire a poussé cette approche encore davantage en se concentrant sur l'organisation, l'autonomisation et le leadership communautaire du développement de programmes. Les travailleurs spécialisés en développement communautaire ont dirigé les membres de la communauté en ciblant les besoins et en exploitant les ressources locales et externes afin d'élaborer des programmes qui répondent à ces besoins. Un groupe du Royaume-Uni a estimé un rendement de l'investissement de $380 \%$ dans le cadre de leur initiative de développement communautaire centrée sur la santé $^{60}$. Les encouragements pour la mobilisation démocratique offerts par les professionnels de la santé peuvent soutenir les communautés marginalisées afin d'accroître leur incidence sur les structures communautaires et les politiques sociales ${ }^{61}$.

Pour qu'une communauté centre ses efforts et réponde aux besoins, un engagement profond envers ses besoins, et leur évaluation, ainsi qu'un soutien pour les efforts menés par la communauté sont nécessaires. Les interventions centrées sur la communauté sont plus facilement mises en œuvre par les grandes équipes interdisciplinaires. Néanmoins, tout professionnel de la santé peut commencer à forger des partenariats avec des organismes communautaires. Aux États-Unis, le programme Health Extension a fourni du financement gouvernemental à 1500 établissements de soins de santé primaires de petite taille pour embaucher des travailleurs afin de répondre aux besoins de la communauté, montrant comment un important donateur peut outiller de petits établissements de soins de santé afin d'approfondir les liens avec leur communautéé ${ }^{2}$.

\section{Comment les équipes de santé peuvent-elles activement s'attaquer aux facteurs structuraux des iniquités au sein de leurs services?}

Un domaine d'enquête émergeant évalue les facteurs d'équité, comme le racisme et le colonialisme ainsi que la manière dont ils se manifestent dans le cadre des interventions des équipes de santé. Les personnes qui sont engagées dans la réponse à ces déterminants de la santé étudient des techniques comme la 
sécurité culturelle, la réflexion critique et les approches antioppressives afin d'aider les professionnels de la santé à comprendre la manière dont les iniquités se manifestent tout au long des interventions de soins de santé, de même qu'à travers les principales structures de notre société63.

À Vancouver, le projet EQUIP explore les effets d'une intervention sur les déterminants structuraux de la santé dans 5 centres de santé communautaires qui desservent les populations socialement marginalisées de l'Ouest du Canada. L'intervention a signalé une amélioration perceptible de la capacité du personnel à reconnaître une iniquité et de répondre aux besoins des clients qui font face à des enjeux médicaux et sociaux complexes ${ }^{64}$. Une évaluation subséquente a montré que les patients rapportaient une amélioration des résultats sur leur santé après l'intervention ${ }^{65}$.

Une initiative pancanadienne sur la sécurité culturelle autochtone a offert une formation en ligne à des milliers de professionnels de la santé66. L'étude de son incidence dans un établissement de soins de santé primaires est présentement en cours ${ }^{67}$.

La participation d'une équipe de santé dans un changement de pratique orienté vers l'équité requiert des ressources pour embaucher et intégrer des spécialistes de l'équité ou pour rechercher une expertise externe qui guidera le processus. II nécessite aussi une volonté de s'attaquer à des sujets qui sont souvent personnellement dérangeants, comme les privilèges des personnes et des groupes sociaux.

\section{Quels sont les défis?}

Malgré les données probantes encourageantes présentées ici, des obstacles à la mise en œuvre de la prescription sociale demeurent. Les approches conventionnelles de l'évaluation des programmes de santé sont, sans doute, mal adaptées pour évaluer les effets des interventions dont on s'attend qu'elles produisent des améliorations à la santé sur une longue période. De plus, les praticiens sont préoccupés, et c'est compréhensible, par le temps et les ressources que ces interventions nécessitent, à la fois pour les instaurer et pour les maintenir ${ }^{68}$. Cependant, des travaux récents ont suggéré que la mobilisation envers les besoins sociaux réduit l'épuisement professionnel des médecins et améliore leur satisfaction au travail de même que la qualité perceptible des soins ${ }^{69-71}$. D'ailleurs, l'importante contribution des conditions sociales dans la détermination de la santé dicte la nécessité impérative d'agir pour y répondre. Il s'agit d'un domaine de pratique émergeant et les professionnels de la santé, les chercheurs et les concepteurs de programmes de santé partagent la responsabilité de la mise en place et de l'évaluation continue des interventions sociales.

Les facteurs sociaux qui nuisent à la santé, comme les iniquités économiques, la misogynie, l'homophobie, le racisme systémique et le colonialisme, requièrent des actions allant audelà du cabinet médical et certaines parties prenantes émettent des mises en garde contre le fait d'essayer de résoudre les besoins sociaux par des soins médicaux, car cela risque de détourner des ressources et des responsabilités du besoin de faire progresser des politiques et de démanteler des structures sociales nuisibles ${ }^{72,73}$.

\section{Conclusion}

Les interventions améliorent la situation sociale des patients en soins de santé primaires et peuvent mener à des améliorations de leur santé et sensibilisent les professionnels de la santé à leur responsabilité de s'attaquer aux conditions sociales nuisibles. Tout professionnel de la santé en soins de santé primaires peut se mobiliser d'une manière ou d'une autre envers l'intervention sociale. L'ampleur et le succès de ces interventions dépendront de la volonté de les prioriser, des ressources qui leur sont attribuées et de la participation des partenaires communautaires et des soutiens multidisciplinaires. Les interventions sociales offrent une importante avancée vers une offre plus holistique de soins de santé primaires.

\section{Références}

1. Kovach KA, Reid K, Grandmont J, et al. How engaged are family physicians in addressing the social determinants of health? A survey supporting the American Academy of Family Physician's health equity environmental scan. Health Equity 2019;3:449-57.

2. Russell G, Dahrouge S, Tuna M, et al. Getting it all done. Organizational factors linked with comprehensive primary care. Fam Pract 2010;27:535-41.

3. Solberg LI. Theory vs practice: should primary care practice take on social determinants of health now? No. Ann Fam Med 2016;14:102-3.

4. Poverty: a clinical tool for primary care providers. Toronto: Centre for Effective Practice (CEP); 2016. Accessible ici : https://cep.health/clinical-products/poverty-a -clinical-tool-for-primary-care-providers/\#pc_page_44 (consulté le 28 janv. 2021).

5. Brcic V, Eberdt C, Kaczorowski J. Corrigendum to "Development of a tool to identify poverty in a family practice setting: a pilot study". Int J Family Med 2015; 2015:418125.

6. Center for Youth Wellness ACEQ \& User Guide. San Francisco: Center for Youth Wellness; 2017. Accessible ici : https://centerforyouthwellness.org/cyw-aceq/ (consulté le 28 janv. 2021).

7. Weir RC, Proser M, Jester M, et al. Collecting social determinants of health data in the clinical setting: findings from national PRAPARE implementation. J Health Care Poor Underserved 2020;31:1018-35.

8. Screening tools comparison table. San Francisco: Social Interventions Research \& Evaluation Network (SIREN), University of San Francisco California. Accessible ici : https://sirenetwork.ucsf.edu/siren-resources/screening-tool -comparison-table-0 (consulté le 28 janv. 2021).

9. Andermann A. Screening for social determinants of health in clinical care: moving from the margins to the mainstream. Public Health Rev 2018;39:19.

10. Tong ST, Liaw WR, Kashiri PL, et al. Clinician experiences with screening for social needs in primary care. J Am Board Fam Med 2018;31:351-63.

11. Gottlieb L, Sandel M, Adler NE. Collecting and applying data on social determinants of health in health care settings. JAMA Intern Med 2013;173:1017-20.

12. Pinto AD, Glattstein-Young G, Mohamed A, et al. Building a foundation to reduce health inequities: routine collection of sociodemographic data in primary care. J Am Board Fam Med 2016;29:348-55.

13. Gottlieb L, Hessler D, Long D, et al. A randomized trial on screening for social determinants of health: the iScreen study. Pediatrics 2014;134:e1611-8.

14. Gold R, Cottrell E, Bunce A, et al. Developing electronic health record (EHR) strategies related to health center patients' social determinants of health. J Am Board Fam Med 2017;30:428-47.

15. Lofters AK, Schuler A, Slater M, et al. Using self-reported data on the social determinants of health in primary care to identify cancer screening disparities: opportunities and challenges. BMC Fam Pract 2017;18:31.

16. Hughes LS, Phillips RL Jr, DeVoe JE, et al. Community vital signs: taking the pulse of the community while caring for patients. J Am Board Fam Med 2016;29:419-22.

17. Ontario Community Health Profiles Partnership [page d'accueil]. Accessible ici : www.ontariohealthprofiles.ca (consulté le 28 janv. 2021).

18. About UTOPIAN. Toronto: University of Toronto; 2021. Accessible ici : https:// www.dfcm.utoronto.ca/about-utopian (consulté le 28 janv. 2021).

19. Kasthurirathne SN, Grannis S, Halverson PK, et al. Precision health-enabled machine learning to identify need for wraparound social services using patient-and population-level data sets: algorithm development and validation. JMIR Med Inform 2020;8:e16129. 
20. Chen S, Bergman D, Miller K, et al. Using applied machine learning to predict healthcare utilization based on socioeconomic determinants of care. Am J Manag Care 2020;26:26-31.

21. Gottlieb LM, Alderwick H. Integrating social and medical care: Could it worsen health and increase inequity? Ann Fam Med 2019;17:77-81.

22. Gottlieb LM, Francis DE, Beck AF. Uses and misuses of patient-and neighborhoodlevel social determinants of health data. Perm J 2018;22:18-078.

23. De Marchis EH, Alderwick H, Gottlieb LM. Do patients want help addressing social risks? J Am Board Fam Med 2020;33:170-5.

24. Rx: Community - Social prescribing in Ontario. Toronto: Alliance for Healthier Communities; 2020. Accessible ici : https://www.allianceon.org/Rx-Community -Social-Prescribing-Ontario (consulté le 28 janv. 2021).

25. Polley M, Fleming J, Anfilogoff T, et al. Making sense of social prescribing. London (UK): University of Westminister; 2017:1-79. Accessible ici : https://42b7de07-529d-4774-b3e1-225090d531bd.filesusr.com/ugd/14f499_81 6dc79e160a4e77991599a74236d0d4.pdf (consulté le 28 janv. 2021).

26. The Social Prescribing Network [page d'accueil]. Accessible ici : https://www. socialprescribingnetwork.com (consulté le 28 janv. 2021).

27. Health Leads [page d'accueil]. Accessible ici : https://healthleadsusa.org (consulté le 28 janv. 2021).

28. Hochman M, Jones A, Doering T, et al. Treating the patient who can't make ends meet. In: Family Medicine Forum; 2019 Oct. 30-Nov. 2; Vancouver. Mississauga (ON): College of Family Physicians Canada.

29. L'Esperance S. Exploring income security offered through primary care: a mixedmethods process evaluation [thesis]. Winnipeg: University of Manitoba; 2019. Accessible ici : hdl.handle.net/1993/34500 (consulté le 28 janv. 2021).

30. Whitelaw S, Thirlwall C, Morrison A, et al. Developing and implementing a social prescribing initiative in primary care: insights into the possibility of normalisation and sustainability from a UK case study. Prim Health Care Res Dev 2017;18:112-21.

31. Gottlieb LM, Wing H, Adler NE. A systematic review of interventions on patients' social and economic needs. Am J Prev Med 2017;53:719-29.

32. Brandling J, House W. Social prescribing in general practice: adding meaning to medicine. Br J Gen Pract 2009;59:454-6.

33. Bertotti $M$, Frostick $C$, Hutt $P$, et al. A realist evaluation of social prescribing: an exploration into the context and mechanisms underpinning a pathway linking primary care with the voluntary sector. Prim Health Care Res Dev 2018;19:232-45.

34. Gottlieb L, Cottrell EK, Park B, et al. Advancing social prescribing with implementation science. J Am Board Fam Med 2018;31:315-21.

35. Alderwick HAJ, Gottlieb LM, Fichtenberg CM, et al. Social prescribing in the US and England: emerging interventions to address patients' social needs. Am J Prev Med 2018;54:715-8

36. Skivington K, Smith M, Chng NR, et al. Delivering a primary care-based social prescribing initiative: a qualitative study of the benefits and challenges. $\mathrm{Br} J \mathrm{Gen}$ Pract 2018;68:e487-94.

37. Adams J, White M, Moffatt S, et al. A systematic review of the health, social and financial impacts of welfare rights advice delivered in healthcare settings. $B M C$ Public Health 2006;6:81.

38. Jones MK, Bloch G, Pinto AD. A novel income security intervention to address poverty in a primary care setting: a retrospective chart review. BMJ Open 2017;7:e014270.

39. Pinto $A D$, Da Ponte $M$, Bondy $M$, et al. Addressing financial strain through a peer-to-peer intervention in primary care. Fam Pract 2020;37:815-20.

40. Black S, Sisco S, Williams $\mathrm{T}$, et al. Return on investment from co-locating tax assistance for low-income persons at clinical sites. JAMA 2020;323:1093-5.

41. Bell ON, Hole MK, Johnson K, et al. Medical-financial partnerships: cross-sector collaborations between medical and financial services to improve health. Acad Pediatr 2020;20:166-74.

42. Sandel M, Hansen M, Kahn R, et al. Medical-legal partnerships: transforming primary care by addressing the legal needs of vulnerable populations. Health Aff (Millwood) 2010;29:1697-705.

43. International Medical-Legal Partnerships. Washington (DC): George Washington University. Accessible ici : https://medical-legalpartnership.org/partnerships/ international/ (consulté le 28 janv. 2021).

44. Klein MD, Beck AF, Henize AW, et al. Doctors and lawyers collaborating to HeLP children: outcomes from a successful partnership between professions. $J$ Health Care Poor Underserved 2013;24:1063-73.

45. Murphy C. Making the case for Medical-Legal Partnerships: an updated review of the evidence, 2013-2020. Washington (DC): George Washington University:1-8. Accessible ici : https://medical-legalpartnership.org/wp-content/ uploads/2020/10/MLP-Literature-Review-2013-2020.pdf (consulté le 28 janv. 2021).

46. Council on Early Childhood; High PC, Klass P. Literacy promotion: an essential component of primary care pediatric practice. Pediatrics 2014;134:404-9.
47. High PC, LaGasse L, Becker S, et al. Literacy promotion in primary care pediatrics: can we make a difference? Pediatrics 2000;105:927-34.

48. Donovan NJ, Blazer D. Social isolation and loneliness in older adults: review and commentary of a National Academies report. Am J Geriatr Psychiatry 2020;28:1233-44.

49. National Academies of Sciences, Engineering, and Medicine; Division of Behavioral and Social Sciences and Education; Health and Medicine Division, et al. Social Isolation and loneliness in older adults: opportunities for the health care system. Washington (DC): The National Academies Press (US); 2020.

50. Veazie S, Gilbert J, Winchell K, et al. Addressing social isolation to improve the health of older adults: a rapid review. Report No. 19-EHC009-EF. Rockville (MD): Agency for Healthcare Research and Quality (US); 2019:1-59. Accessible ici : https://www.ncbi.nlm.nih.gov/sites/books/NBK537909/pdf/Bookshelf_ NBK537909.pdf (consulté le 28 janv. 2021).

51. Pinto AD, Hassen N, Craig-Neil A. Employment interventions in health settings: a systematic review and synthesis. Ann Fam Med 2018;16:447-60.

52. Aubry $\mathrm{T}$, Bloch $\mathrm{G}$, Brcic V, et al. Effectiveness of permanent supportive housing and income assistance interventions for homeless individuals in high-income countries: a systematic review. Lancet Public Health 2020;5:e342-60.

53. Solomon EM, Wing $\mathrm{H}$, Steiner JF, et al. Impact of transportation interventions on health care outcomes: a systematic review. Med Care 2020;58:384-91.

54. Beck AF, Henize AW, Kahn RS, et al. Forging a pediatric primary care-community partnership to support food-insecure families. Pediatrics 2014;134:e564-71.

55. Tarasuk V, Vogt J. Household food insecurity in Ontario. Can J Public Health 2009;100:184-8.

56. De Marchis EH, Torres JM, Benesch $\mathrm{T}$, et al. Interventions addressing food insecurity in health care settings: a systematic review. Ann Fam Med 2019;17:436-47.

57. Henize AW, Beck AF, Klein MD, et al. A road map to address the social deter minants of health through community collaboration. Pediatrics 2015;136: e993-1001.

58. Tsega M, Lewis C, McCarthy D, et al. Review of evidence on the health care impacts of interventions to address the social determinants of health. New York: The Commonwealth Fund; 2019. Accessible ici : https://www.commonwealth fund.org/sites/default/files/2019-07/COMBINED_ROI_EVIDENCE_REVIEW_7.15. 19.pdf (consulté le 28 janv. 2021).

59. Gunderson JM, Wieland ML, Quirindongo-Cedeno O, et al. Community health workers as an extension of care coordination in primary care: a communitybased cosupervisory model. J Ambul Care Manage 2018;41:333-40.

60. Fisher B. Community development through health gain and service change: Do it now! London J Prim Care (Abingdon) 2014;6:154-8.

61. Raza D, Brown C, Pinto AD. Supporting patients to shape social determinants of health through democratic engagement. Can Fam Physician 2020;66:639-41.

62. Fagnan LJ. Moving upstream: health extension and primary care. J Am Board Fam Med 2017;30:10-2.

63. Browne AJ, Varcoe CM, Wong ST, et al. Closing the health equity gap: evidence based strategies for primary health care organizations. Int J Equity Health 2012;11:59.

64. Browne AJ, Varcoe C, Ford-Gilboe M, et al. Disruption as opportunity: impacts of an organizational health equity intervention in primary care clinics. Int $J$ Equity Health 2018;17:154.

65. Ford-Gilboe M, Wathen CN, Varcoe C, et al. Annette J Browne For The Equip Research Program. How equity-oriented health care affects health: Key mechanisms and implications for primary health care practice and policy. Milbank Q 2018;96:635-71.

66. San'yas Indigenous Cultural Safety Training [page d'accueil]. Vancouver: Provincial Health Services Authority. Accessible ici : www.sanyas.ca/home (consulté le 28 janv. 2021).

67. Reconciling relationships. Toronto: Centre for Urban Health Solutions (C-UHS). Accessible ici : www.welllivinghouse.com/what-we-do/projects/reconciling -relationships/ (consulté le 28 janv. 2021).

68. Bloch G, Rozmovits L, Giambrone B. Barriers to primary care responsiveness to poverty as a risk factor for health. BMC Fam Pract 2011;12:62.

69. Kung A, Cheung T, Knox M, et al. Capacity to address social needs affects primary care clinician burnout. Ann Fam Med 2019;17:487-94.

70. De Marchis E, Knox M, Hessler D, et al. Physician burnout and higher clinic capacity to address patients' social needs. J Am Board Fam Med 2019;32:69-78.

71. Pantell MS, De Marchis E, Bueno A, et al. Practice capacity to address patients social needs and physician satisfaction and perceived quality of care. Ann Fam Med 2019;17:42-5.

72. Maani N, Galea S. The role of physicians in addressing social determinants of health. JAMA 2020;323:1551-2.

73. Mackenzie M, Skivington K, Fergie G. "The state They're in": unpicking fantasy paradigms of health improvement interventions as tools for addressing health inequalities. Soc Sci Med 2020;256:113047. 
Intérêts concurrents : Gary Bloch a obtenu une subvention de recherche du Collège des médecins de famille du Canada et de l'Institut Wellesley ainsi que des honoraires de conférencier provenant de départements universitaires en médecine familiale, du Collège des médecins de famille du Canada, de collèges provinciaux de médecins de famille et du Réseau des hôpitaux faisant la promotion de la santé au Japon (Japan Health Promoting Hospitals Network), indépendamment des travaux soumis. Il est membre du comité directeur de l'organisme Defend Disability. Linda Rozmovits est une consultante indépendante qui a été rémunérée à même les fonds de recherche pour participer aux travaux soumis.

Cet article a été révisé par des pairs.

Affiliations : Département de médecine familiale et communautaire (Bloch), Université de Toronto; Service de médecine familiale et communautaire (Bloch), Hôpital St. Michael; Groupe Inner City Health Associates (Bloch); consultante indépendante en recherche qualitative sur la santé (Rozmovits), Toronto, Ont.

Collaborateurs : Gary Bloch a dirigé la conception du travail. Les deux auteurs ont participé à la conception de la revue documentaire, à la réalisation de la revue documentaire et à son analyse ainsi qu'à la rédaction, à l'édition et à la finalisation du manuscrit. Les deux auteurs endossent l'entière responsabilité de tous les aspects du travail.

Soutien financier : L'élaboration de cet article a été soutenue grâce à du financement offert par la Fondation AMS, le Service de médecine familiale et communautaire de l'Hôpital St. Michael, le Département de médecine familiale et communautaire de l'Université de Toronto et le laboratoire d'innovation Health Commons Solutions Lab.

Propriété intellectuelle du contenu : Il s'agit d'un article en libre accès distribué conformément aux modalités de la licence Creative Commons Attribution (CC BY-NC-ND 4.0), qui permet l'utilisation, la diffusion et la reproduction de tout médium à la condition que la publication originale soit adéquatement citée, que l'utilisation se fasse à des fins non commerciales (c.-à-d., recherche ou éducation) et qu'aucune modification ni adaptation n'y soit apportée. Voir : https://creativecommons.org/licenses/by-nc-nd/4.0/deed.fr.

Correspondance : Gary Bloch, gary.bloch@utoronto.ca 